\title{
ПРИМЕНЕНИЕ СИСТЕМНОГО АНАЛИЗА РАСПРЕДЕЛЕННЫХ СИНГУЛЯРНОВОЗМУЩЕННЫХ СИСТЕМ ОПТИМАЛЬНОГО УПРАВЛЕНИЯ ДЛЯ ПРОЦЕССОВ С ТЕПЛОПЕРЕДАЧЕЙ, МАССОПЕРЕДАЧЕЙ, ХИМИЧЕСКИМИ РЕАКЦИЯМИ
}

\section{THE ANALYSIS OF DISTRIBUTED SINGULARLY PERTURBED OPTIMAL CONTROL SYSTEMS USE FOR PROCESSES WITH HEAT TRANSFER, MASS TRANSFER, CHEMICAL REACTIONS}

M. Kovaleva

Summary. The article discusses the issue of modeling systems with distributed parameters for processes with heat transfer, mass transfer, chemical reactions. In the modern world, great attention is paid to the development of all spheres of social life, a lot of efforts are spent to accelerate technological progress and use numerous innovations. But in order to achieve success in this, sometimes it is necessary to combine the incompatible, and most importantly, be able to evaluate your own work with the help of an experiment.

It is shown in the article that, along with lumped problems, one can develop a regularization method applicable to distributed singularly perturbed optimal control problems.

Keywords: the system analysis, experiment, management, singular indignant.

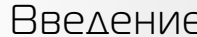

B сложной системе обычно присутствуют два уровня управления. В медленно меняющейся обстановке децентрализованная часть системы успешно справляется с адаптацией поведения системы к среде и с достижением глобальной цели системы за счет оперативного управления, а при резких изменениях среды осуществляется централизованное управление по переводу системы в новое состояние. Недостатком централизованного управления является сложность управления из-за огромного потока информации, подлежащей переработке в системе управления [1].

В современном мире огромное внимание уделяется развитию всех сфер общественной жизни, тратится множество усилий для ускорения технического прогресса и использования многочисленных инноваций. Но для
Ковалева Мария Александровна

Дочент, Владикавказский филиал ФГОБУ ВО «Финансовый университет при правительстве РФ» mary_kovaleva@list.ru

Аннотация. В статье рассматривается вопрос моделирования систем с распределенными параметрами для процессов с теплопередачей, массопередачей, химическими реакциями. В современном мире огромное внимание уделяется развитию всех сфер общественной жизни, тратится множество усилий для ускорения технического прогресса и использования многочисленных инноваций. Но для того, чтобы добиться в этом успеха, порой необходимо сочетать не сочетаемое, а главное уметь при помощи эксперимента оценить собственный труд.

В статье показывается, что наряду с сосредоточенными задачами можно развить метод регуляризации, применимый к распределенным сингулярновозмущенным задачам оптимального управления.

Ключевые слова: системный анализ, эксперимент, управление, сингулярновозмущенный.

того, чтобы добиться в этом успеха, порой необходимо сочетать не сочетаемое, а главное уметь при помощи эксперимента оценить собственный труд. В настоящее время системный подход в экспериментальных исследованиях является наиболее эффективным способом получения данных. Данная тема является интересной и перспективной, ведь системный анализ используется в экономическом мире ежедневно [10].

Сложные открытые системы, к которым можно отнести технологические процессы, не подчиняются вероятностным законам. Такие системы можно назвать сингулярновозмущенными.

\section{Постановка заАачи}

Рассмотрим вопрос моделирования систем с распределенными параметрами, в случае процессов при нали- 
чии, теплопередачи, массопередачи, химических реакций (основных технологических процессов, в частности в металлургии) [1-9].

Оптимизация тепло и массопереноса в металлургических агрегатах

Учитывая выражение $J_{k}=-D \rho$ получим обобщенное дифференциальное уравнение диффузии.

$$
\rho \frac{d m_{k}}{d \tau}=\left[D \rho\left(\operatorname{gradm}_{k}+\frac{\delta_{p}}{p}+k_{T} / T \operatorname{grad} T\right)\right]
$$

$M_{k} \sum_{r=1}^{R} \gamma_{k r} W_{r}$, где $\mathrm{k}=1,2, . \mathrm{N}-1$ (1)

Далее, запишем уравнение неразрывности

$$
\frac{d \rho}{d \tau}+\operatorname{div}(\rho \vartheta=0
$$

или в другом виде:

$$
\frac{d \rho}{d \tau}+\rho \operatorname{div}(\vartheta)=0
$$

$\vartheta$ - скорость центра масс, $\rho$ - общая плотность. Рассмотрим коэффициенты, стоящие перед градиентами, постоянными (не зависящими от координат), тогда из (2) получим

$$
\rho \frac{d m_{k}}{d \tau}=D \rho\left(\nabla^{2} m_{k}+\frac{\delta_{p}}{P \nabla^{2} \mathrm{c}}+\frac{k_{T}}{T \nabla^{2} T}\right)+M_{k} \sum_{r=1}^{R} \Gamma_{k r} W_{r},
$$

$(k=1,2 \cdot N-1)$.

Далее, составим уравнение переноса импульса. Учитывая, что $\operatorname{div}($ д $=\operatorname{grad} P$ получим уравнение Навье.

$$
\begin{aligned}
& \mathrm{c} \frac{d \vartheta}{d \Phi}=-\operatorname{gradP}+\operatorname{Div}[\nabla и \dot{ }+\nabla и \dot{~}-2 / 3 \text { з(и́)д }]+ \\
& +\sum_{k=1}^{N} \mathrm{c}_{k} \cdot K_{k}^{\prime}
\end{aligned}
$$

д - единичный тензор второго ранга, компонентами которого являются символы Кронекера д $_{i j}$.Уравнение (5) используется как составная часть математической модели металлургического агрегата в тех случаях, когда в исследуемой области весьма существенна неравномерность температурного поля или когда значительны эффекты турбулентного потока. Например, при факельном отоплении печи. Для потоков с развитой турбулентностью коэффициент динамической молекулярной вязкости $\eta$ заменяют его эффективным значением. $3_{э \phi}=3+3_{T}$. Здесь $3_{T}$ коэффициент динамической турбулентной (вихревой) вязкости. При моделировании высокотемпературных печей камерного типа (особенно печей с постоянной температурой рабочего пространства можно полагать $\eta=$ const. Тогда, учитывая, что ( $\nabla$ и́) $=\operatorname{grad}(\operatorname{div}$ и) из (5) находим уравнения Навье-Стокса для сжимаемой жидкости с постоянной вязкостью. $\mathrm{c} \frac{d \dot{\vartheta}}{d \phi}=-\nabla P+3 \nabla^{2} \hat{\vartheta}_{+} \frac{1}{3} \nabla(\nabla \hat{\vartheta})+\sum_{k=1}^{N} c_{k} K_{k}^{\prime}$

В случае, когда внешние силы обусловлены лишь одной градацией, тогда

$$
\sum_{k=1}^{N} \mathrm{c}_{k} K_{k}^{\prime}=\mathrm{c} g
$$

Далее, рассмотрим уравнение баланса внутренней энергии.

$$
\mathrm{c} \frac{d u}{d \Phi}=-J_{U}^{\prime}+\mathrm{q}(\mathrm{U})
$$

Здесь $I_{U}^{\prime}$ называют неконвективным потоком энергии. Из (8), после ряда преобразований получаем дифференциальное уравнение Фурье - Кирхгофа.

Допустим, это будет одна из зон нагревательной печи или самостоятельная нагревательная печь следующей конструкции. Ограничимся анализом процессов в контрольном объеме, показанном штриховой линией. Основным энергоносителем в данном агрегате является топливо (продукты его сгорания). Система химических реакций, которые возникают при горении, весьма сложна. Учитывая сказанное, поступим следующим образом. Будем считать топливо и окислитель простыми реагентами, которые всегда соединяются в одном и том же (стехиометрическом) соотношении. Тогда обозначая индексом «гор» и «ок» соответственно топливо (горючее) и окислитель, можно записать: 1 кг горючего і кг окислителя $\rightarrow(1+\mathrm{i})$ кг продуктов сгорания. Если $R_{\text {гор }}$ и $R_{\text {ок }}$ обозначить скорости расходования горючего и окислителя в процессе химической реакции $R_{j}=M_{j} \Gamma_{j} W_{i}$, то данное предложение сводится к соотношению $R_{\text {гор }}=R_{\text {ок }} / \mathrm{i}$.

Как правило, изменение давления в рабочем пространстве нагревательных печей весьма мало, так что без существенной потери точности допустимо полагать $\operatorname{gradP}=0$. Учитывая, что для газов в области температур до $2500 \mathrm{~K} k_{T} 10^{-3}-10^{-5}$, можно пренебречь и термодиффузией. В таком случае (2) значительно упростится и примет вид.

$$
\begin{aligned}
& \mathrm{C}_{+\mathrm{И}_{z}} \frac{\partial m_{\text {гор }}}{\partial z}=\frac{\partial}{\partial x}\left(D_{э ф г о р} \mathrm{C} \frac{\partial m_{\text {гор }}}{\partial x}\right)+ \\
& +\frac{\partial}{\partial z}\left(D_{э \phi г о р} \mathrm{C} \frac{\partial m_{\text {гор }}}{\partial z}\right)+R_{\text {гор }}
\end{aligned}
$$

Аналогично получим уравнение для $m_{\text {ок. }} D_{э \phi}-э \phi-$ фективный коэффициент диффузии, свой для ламинарного и турбулентного режимов течения, $R_{\text {гор }}=-K_{p}^{2} m_{\text {гор }} m_{\text {ок }}$ $\exp [-E /(R T)]$.

$E$ и К эмпирические постоянные, которые определяются видом топлива и типом топливо сжигающе- 
го устройства. Объединив уравнение неразрывности с уравнением (9), получим.

$$
\begin{aligned}
& \frac{\partial\left(\mathrm{cm}_{j}\right)}{\partial \phi}+\frac{\partial\left(\mathrm{cu}_{x} m_{j}\right)}{\partial x}+\frac{\partial\left(\mathrm{cu}_{z} m_{j}\right)}{\partial z}= \\
& =\frac{\partial\left(D_{3 \phi j} \mathrm{cu}_{x} m_{j}\right)}{\partial x}+\frac{\partial\left(\mathrm{cu}_{z} m_{j}\right)}{\partial z}+R_{j}
\end{aligned}
$$

(где j= гор, ок).

Это первое уравнение модели рассматриваемого агрегата, оно записано в консервативной или дивергентной форме, т.е. в форме закона сохранения.

Запишем уравнение (10) в векторной форме:

$$
c_{p}\left[\frac{\partial\left(\mathrm{cm}_{j}\right)}{\partial t}+\left(\text { си́m } m_{j}\right)\right]=\left(\pi_{э \Phi} \operatorname{gradm}_{j}\right)+R_{j}
$$

Рассмотрим далее, уравнения движения (уравнения баланса импульса). Объединяя уравнения (11) с общим уравнением неразрывности, можно получить:

$$
\begin{aligned}
& \frac{\partial\left(\mathrm{cu}_{x}\right)}{\partial t}+\frac{\partial\left(\mathrm{cu}_{x}^{2}\right)}{\partial x}+\frac{\partial\left(\mathrm{cu}_{z} \mathrm{H}_{x}\right)}{\partial z}=\frac{-\partial p}{\partial x}+\partial+\left(\frac{1}{3} \partial\right. \\
& \frac{\partial\left(\mathrm{cu}_{z}\right)}{\partial t}+\frac{\partial\left(\mathrm{cu}_{Z}^{2}\right)}{\partial x}+\frac{\partial\left(\mathrm{cu}_{z} \mathrm{H}_{x}\right)}{\partial z}=\frac{-\partial p}{\partial z}+\partial+\left(\frac{1}{3} \partial\right.
\end{aligned}
$$

Рассмотрим далее, каноническую форму уравнений (12), (13):

$$
\begin{aligned}
& \left.\frac{\partial\left(\mathrm{cu}_{x}\right)}{\partial t}+\left(\text { си́и }{ }_{x}\right)=+\right)+R_{\mathrm{u}_{x}} \\
& \frac{\partial\left(\mathrm{cu}_{z}\right)}{\partial t}+\operatorname{div}\left(\text { си́и }{ }_{z}=\right)+R_{\mathrm{u}_{z}} \\
& \left.R_{\mathrm{u}_{x}}=\frac{-\partial}{\partial x}+(1 / 3) \partial\right) / \partial x \\
& \left.R_{\mathrm{u}_{z}}=\frac{-\partial}{\partial z}+(1 / 3) \partial\right) / \partial z
\end{aligned}
$$

Далее, рассмотрим уравнение Фурье - Кирхгофа. Для условий работы рассматриваемого агрегата, как уже говорилось, будем считать градиент давления крайне малым, поэтому термоэффектом вызванным давлением будем пренебрегать.

Будем пренебрегать работой сил давления и аэродинамического нагрева, переносом теплоты за счет диффузии под действием внешних сил (сил тяжести), а также диффузионного термоэффекта. Учитывая данные обстоятельства, упростим уравнение энергии. Учитывая сказано выше, запишем уравнение энергии, включающее общее уравнение неразрывности:

$$
\begin{aligned}
& C_{p}\left[\frac{\partial(c T)}{\partial t}+\frac{\partial\left(n_{x} T\right)}{\partial x}+\frac{\partial\left(n_{z} T\right)}{\partial z}\right]= \\
& =\frac{\partial\left(\pi_{3 \phi} \partial T / \partial x\right)}{\partial x}+\frac{\partial\left(\pi_{3 \phi} \partial T / \partial z\right)}{\partial z}+R_{t}
\end{aligned}
$$

Здесь $R_{t}=-\sum_{j=1}^{2} H_{j} R_{j}+$

$$
\begin{aligned}
& +\frac{\partial}{\partial x\left[\frac{{ }_{3}^{3} \phi\left(\frac{\mathrm{n}^{2}}{2}\right)}{\partial x}+\frac{\Gamma_{k} \partial E_{t}}{\partial x}+\frac{c \sum_{j=1}^{2} Q_{j} D_{3 \phi j} \partial m_{j}}{\partial x}\right]}+ \\
& +\frac{+\partial\left[\frac{{ }_{3{ } \phi} \partial\left(\frac{\mathrm{u}^{2}}{2}\right)}{\partial z}+\frac{\Gamma_{k} \partial E_{t}}{\partial z}+\frac{c \sum_{j=1}^{2} Q_{j} D_{3 \phi j} \partial m_{j}}{\partial z}\right]}{\partial z}
\end{aligned}
$$

$-\sum_{j=1}^{2} c_{p_{j}} J_{i}^{\prime} \operatorname{gradT}$.

$E_{t}=\left[\left(\mathrm{u}_{x}\right)^{2}+\left(\mathrm{u}_{y}\right)^{2}+\left(\mathrm{u}_{z}\right)^{2}\right] / 2 ; F_{k}=+3_{\mathrm{TyP}} /\left(\mathrm{cy}_{E}\right)$ коэффициент переноса (диффузии) энергии турбулентности. В векторной форме уравнения запишется следующим образом

$$
\mathrm{c}_{p}\left[\frac{\partial(\mathrm{c} T)}{\partial t}+(\mathrm{cú} T)\right]=\left(\pi_{э ф} \operatorname{grad} T\right)+R_{t}
$$

Далее, запишем уравнение турбулентных пульсаций

$$
\partial /\left(\mathrm{c} E_{t}\right) / \partial t+\left(\mathrm{c} E_{\phi} \mathrm{u}\right)=\left(\Gamma_{k} \operatorname{grad} E_{\phi}\right)+G-\mathrm{ce}
$$

где е - скорость диссипации (рассеяния) энергии турбулентности, а G скорость генерации этой энергии. Аналогичное дифференциальное уравнение записывается для переменной е.

Нетрудно заметить, что все записанные ранее уравнения однотипны. Обозначим искомую функцию Ф, тогда всю систему уравнений, рассмотренную выше можно записать в виде одного обобщенного выражения.

$$
\begin{aligned}
& a_{\phi}\left[\frac{\partial \Phi}{\partial \phi}+(\text { си́ } \Phi)\right]=\left(b_{\Phi} \operatorname{grad}_{\Phi}\right)+D_{\Phi}, \\
& \text { где } a_{\Phi}=\left\{1,1,1,1, C_{P}, 1,1\right\}^{T}-
\end{aligned}
$$

вектор столбец переменных параметров системы;

$$
\Phi=\left\{m_{\text {rop }}, m_{\text {ок }}, \mathrm{u}_{x}, \mathrm{u}_{z}, T, E_{T}, \mathrm{e}\right\}^{T}-
$$

вектор-столбец исходных функций;

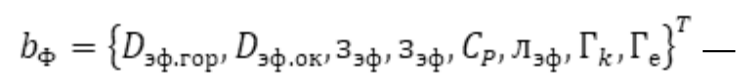

вектор столбец коэффициентов переноса и

$d_{\Phi}=\left\{R_{\text {гор }}, R_{\text {ок }}, R_{\mathrm{u}_{x}}, R_{\mathrm{u}_{z}}, R_{t}, R_{E}, R_{\mathrm{e}}\right\}^{T}$.

Уравнение (20) пригодно для дальнейшего анализа и разработки моделей систем оптимального управления.

Учитывая щ для двумерного течения

$щ=\frac{\partial u_{x}}{\partial z}-\partial u_{z} / \partial x$ 
после некоторых преобразований придем к уравнению:

$$
\begin{aligned}
& \frac{\partial(\mathrm{cm})}{\partial t}+\frac{\partial\left(\mathrm{cn}_{x}\right. \text { щ) }}{\partial x}+\frac{\partial\left(\mathrm{cn}_{z}\right. \text { щ) }}{\partial z}= \\
& =\frac{\partial\left(3_{3 \phi} \partial щ / \partial x\right)}{\partial x}+\frac{\partial\left(3_{3 \phi} \partial щ / \partial z\right)}{\partial z}+R_{\text {щ' }}
\end{aligned}
$$

где

$$
\begin{aligned}
& R_{\text {щ }}=, Q_{\text {щ }}=\left(\frac{\partial 3_{3 \phi}}{\partial z}\right) \nabla^{2} \mathrm{и}_{x}--\left(\frac{\partial 3_{3 \phi}}{\partial x}\right) \nabla^{2} \mathrm{и}_{z}- \\
& -\left(\frac{\partial^{2}{3_{3 \phi}}}{\partial x \partial z}-\partial \mathrm{u}_{z} / \partial z\right)-/ \partial z \text {. }
\end{aligned}
$$

Поскольку $Q_{\text {щ }}$ зависит от эффективной вязкости, а ее изменение в пространстве не может быть описано достаточно точно, обычно полагают $Q_{\text {щ }}=0$.

Очень важной задачей математического моделирования металлургических агрегатов является установление оптимальных стационарных режимов работы. Отклонение от этих режимов всегда можно описать более простыми методами, используя процедуру линериализации уравнений.

Поэтому учитывая стационарное уравнение неразрывности

$$
\frac{\partial\left(\mathrm{ch}_{x}\right)}{\partial x}+\frac{\partial\left(\mathrm{CH}_{z}\right)}{\partial z}=0
$$

введем функцию тока $\psi(x, z)$ при помощи равенств

$$
\mathrm{Cu}_{x}=\frac{\partial \mathrm{m}}{\partial z}, \mathrm{Cu}_{z}=\frac{-\partial \mathrm{m}}{\partial x} \mathrm{M} .1
$$

Тогда уравнение примет вид:

$\partial\left(m_{j} \frac{\partial \mathrm{w}}{\partial z}\right) / \partial x-\frac{\partial\left(m_{j} \frac{\partial \mathrm{w}}{\partial x}\right)}{\partial z}=$

$\left.=\partial\left(D_{э ф j} \mathrm{c} \partial m_{j}\right) / \partial \mathrm{x} / \partial x+\partial / \partial \mathrm{z}\right) / \partial \mathrm{z}+R_{j} \mathrm{M} .2$

(j=гор, ок)

И соответственно

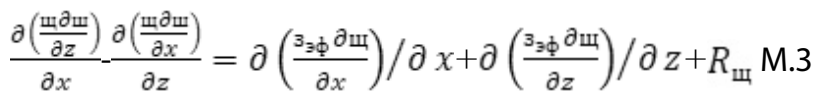

Учитывая М1 в формуле для потока

$\frac{\partial \mathrm{u}_{x}}{\partial z}-\partial \mathrm{u}_{z} / \partial x$

найдем уравнение для нахождения $\psi$.

$\frac{\partial\left(\frac{1}{\frac{c \partial w}{\partial x}}\right)}{\partial x}+\frac{\partial\left(\frac{1}{\frac{c \partial w}{\partial z}}\right)}{\partial z}=$ щ M.4

Наконец, учитывая уравнение энергии, получим.

$$
C_{p}\left[\frac{\partial\left(\frac{T \partial \mathrm{w}}{\partial z}\right)}{\partial x}-\frac{\partial\left(\frac{T \partial \mathrm{w}}{\partial x}\right)}{\partial z}\right]=\frac{\partial\left(\frac{\pi_{3 \phi} \partial T}{\partial x}\right)}{\partial x}+\frac{\partial\left(\frac{\pi_{3 \phi} \partial T}{\partial z}\right)}{\partial z}+R_{T}
$$

Учитывая, что уравнения М.2- М.5 однотипны, запишем их в векторной форме.

$$
C_{p}\left[\frac{\partial\left(\frac{\Phi \partial w}{\partial z}\right)}{\partial x}-\frac{\partial\left(\frac{\Phi \partial w}{\partial x}\right)}{\partial z}\right]=\frac{\partial\left(\frac{b_{3 \phi} \partial T}{\partial x}\right)}{\partial x}+\frac{\partial\left(\frac{b_{3 \phi} \partial T}{\partial z}\right)}{\partial z}+\Phi_{T}
$$

Уравнение в квадратных скобках описывает диффузионный перенос, а последнее слагаемое отражает образование параметра источниками (химическими реакциями).

Далее, осталось описать нагревание обрабатываемых изделий и ограничиваемых стенок.

Здесь мы можем использовать для изделий уравнение теплопроводности. Пусть $T_{1}$ - температура металла, перемещение изделия в сторону начала координат $и_{\mathrm{M}}$. Запишем уравнение теплопроводности.

$$
\frac{-\mathrm{C}_{M} \mathrm{c}_{M} \mathrm{~h}_{M} \partial T_{1}}{\partial x}=\partial\left(\frac{\pi_{M} \partial T_{1}}{\partial x}\right) \partial x+\partial\left(\frac{\pi_{M} \partial T_{1}}{\partial z}\right) \partial z \text { M.6 }
$$

Граничные условия

$$
\begin{aligned}
& z=0, \frac{\partial T_{1}}{\partial z}=0 ; \\
& z=S, \\
& T_{1}(x)=T(x) ; \pi_{M}\left(\partial T_{1} / \partial z\right)=\pi_{э \Phi}\left(\partial T_{1} / \partial x\right)+q_{R z}, \\
& S-\text { полутолщина изделия. }
\end{aligned}
$$

Для ограждающих стенок справедливо стационарное уравнение теплопроводности, причем локально одномерное (теплота распространяется по нормали к стенке). На внутренней поверхности стенки выполняются граничные условия сопряжения (четвертого рода). На наружной стенке - граничные условия третьего рода (конвективный теплообмен с окружающей средой).

Система уравнений (М.1)-(М.6) не исчерпывает всего многообразия явлений, развивающемся в рабочем пространстве моделируемого агрегата.

При внимательном анализе может возникнуть необходимость в добавлении к указанной системе других соотношений, в частности, для термодинамических и переносных свойств веществ. Однако можно утверждать, что уравнения (М.1)-(М.6) учитывают, основные, главные стороны процесса, протекающие в рассматриваемом агрегате. 
Далее, еще раз отметим, что целью здесь ставится не получение уравнений математической модели какого-либо конкретного явления. Основные результаты сводятся к следующему:

1. При разработке конкретных моделей металлургических объектов очень важно выявить и учесть взаимовлияние разных явлений, составляющих данный процесс.

2. Уравнения, описывающие разные явления (разные аспекты процессов тепло - массопереноса), имеют одинаковую структуру или могут быть сведены к форме с данной структурой.

Запишем уравнения состояния металлургического агрегата, как было показано выше, эти уравнения имеют следующий вид:

$$
\begin{aligned}
& \frac{\partial\left(\mathrm{cm}_{j}\right)}{\partial t}+\frac{\partial\left(\mathrm{cu}_{x} m_{j}\right)}{\partial x}+\frac{\partial\left(\mathrm{cn}_{z} m_{j}\right)}{\partial z}= \\
& =\frac{\partial\left(D_{3 \phi} \mathrm{cu}_{x} m_{j}\right)}{\partial x}+\frac{\partial\left(\mathrm{cn}_{z} m_{j}\right)}{\partial z}+R_{j},
\end{aligned}
$$

(где $\mathrm{j}=$ гор, ок).

$$
\begin{aligned}
& \left.\frac{\partial\left(\mathrm{cи}_{x}\right)}{\partial t}+\left(\text { си́и }_{x}\right)=+\right)+R_{\mathrm{n}_{x^{\prime}}} \\
& \frac{\partial\left(\mathrm{cu}_{z}\right)}{\partial t}+\operatorname{div}\left(\text { си́ }_{z}=\right)+R_{\mathrm{n}_{z^{\prime}}}
\end{aligned}
$$

$\left.\left.R_{\mathrm{n}_{x}}=\frac{-\partial p}{\partial x}+(1 / 3) \partial\right) / \partial x, R_{\mathrm{n}_{z}}=\frac{-\partial p}{\partial z}+(1 / 3) \partial\right) / \partial z$

$\mathrm{c}_{p}\left[\frac{\partial(\mathrm{c} T)}{\partial t}+(\right.$ си́ $\left.T)\right]=\left(л_{э \phi} \operatorname{grad} T\right)+R_{t}$

$\partial /\left(\mathrm{c} E_{t}\right) / \partial t+\left(\mathrm{c} E_{t} \mathrm{u}\right)=\left(\Gamma_{k} \operatorname{grad} E_{t}\right)+G-\mathrm{ce}$

Уравнения (24)-(27), которое зависит от интенсивности источников химических реакций. Интенсивностью этих источников определяется характер состояния процессов, протекающих в металлургических агрегатах.

Пусть интенсивностьхимическихисточниковзадается следующим набором параметров $u=u_{2}, \ldots, u_{r}$. В таком случае можно записать $R_{j}=R_{j}(u, t), R_{\mathrm{n}_{x}}=R_{\mathrm{n}_{x}}(u, t)$, $R_{\mathrm{n}_{z}}=R_{\mathrm{n}_{z}}(u, t), R_{t}=R_{t}(u, t)$.

Таким образом, изменяя интенсивность источников химических реакций, мы можем влиять на состояние металлургического агрегата, т.е. управлять данным агрегатом. Естественно, $u_{i}, i=1,2, \ldots, r$ должны подчиняться некоторым ограничениям, например $\left|u_{i}\right| \leq \Gamma_{i}$, которые определяют область управления задачи. Если теперь еще доб ть некоторый минимизируемый функционал.

$$
J=J\left(m_{j} . \mathrm{и}_{x}, \mathrm{и}_{z}, T, u, t\right)
$$

Тогда получим некоторую задачу оптимального управления (24-28) для металлургического агрегата.
Нетрудно видеть, что данная задача будет 3 сеть от параметров: $D_{э ф, j}, 3_{э ф}, \pi_{э ф}$ и если хотя бы один из них является малым, тогда поставленная задача оптимального управления будет сингулярновозмущенной.

Рассмотрим первоначально однопараметрический случай. Допустим $3_{э ф}=\pi \rightarrow 0$. Здесь мы сталкиваемся с сингулярной задачей оптимального управления. Так же, как это мы делали выше, для регуляризации этой задачи применим метод Уизема.

Перепишем уравнения (24-28) следующим образом:

$$
\begin{aligned}
& \frac{\partial\left(\mathrm{cm}_{j}\right)}{\partial t}+\frac{\partial\left(\mathrm{cm}_{j} \mathrm{n}_{x}\right)}{\partial x}+\frac{\partial\left(\mathrm{cm}_{j} \mathrm{n}_{z}\right)}{\partial z}= \\
& \frac{\partial\left(D_{3 \phi j} \mathrm{c} \frac{\partial m_{j}}{\partial x}\right)}{\partial x}+\frac{\partial\left(D_{3 \phi j} \mathrm{c} \frac{\partial m_{j}}{\partial z}\right)}{\partial z}+R_{j} \\
& \frac{\partial\left(\mathrm{cu}_{x}\right)}{\partial t}+\frac{\partial\left(\mathrm{cu}_{x}^{2}\right)}{\partial x}+\frac{\partial\left(\mathrm{cn}_{x} \mathrm{u}_{z}\right)}{\partial z}= \\
& -\frac{\partial P}{\partial x}+\frac{\partial\left(3_{3 \phi} \frac{\partial u x}{\partial x}\right)}{\partial x}+\frac{\partial\left(3_{3 \phi} \frac{\partial u x}{\partial z}\right)}{\partial z}+\frac{\frac{1}{3} \partial\left(3_{3 \phi} \div \hat{n}\right)}{\partial x}, \\
& c_{p}\left[\frac{\partial(\mathrm{c} T)}{\partial t}+\frac{\partial\left(\mathrm{cu}_{x} T\right)}{\partial x}+\frac{\partial\left(\mathrm{cu}_{z} T\right)}{\partial z}\right]= \\
& =\frac{\partial\left(s_{3 \phi} \frac{\partial u z}{\partial x}\right)}{\partial x}+\frac{\partial\left(s_{3 \phi} \frac{\partial u z}{\partial z}\right)}{\partial z}+R_{t}
\end{aligned}
$$

Допустим, имеем следующие асимптотические разложения:

$$
\begin{aligned}
& \left.\left.\left.\mathrm{u}_{x}, \mathrm{z}, \mathrm{\eta}, \mathrm{t}\right)=\mathrm{u}_{x 0}, \mathrm{z}, \mathrm{t}\right)+\mathrm{u}_{x 1}, \mathrm{z}, \mathrm{t}\right) 3+ \\
& \left.+\mathrm{u}_{x 2}, \mathrm{z}, \mathrm{t}\right)\left(3^{2}+, \ldots . .+\mathrm{o}\left(3^{k}\right.\right. \\
& \left.\left.\left.\mathrm{u}_{z}, \mathrm{z}, \mathrm{\eta}, \mathrm{t}\right)=\mathrm{u}_{z 0}, \mathrm{z}, \mathrm{t}\right)+\mathrm{u}_{z 1}, \mathrm{z}, \mathrm{t}\right) 3+ \\
& +\mathrm{u}_{z 2}\left(\phi, x, z, t 3^{2}+, \ldots \ldots+, \mathrm{o}\left(3^{k}\right.\right. \\
& \left.\left.T, \mathrm{z}, \eta, \mathrm{t})=T_{0}, \mathrm{z}, \mathrm{t}\right)+T_{1}, \mathrm{z}, \mathrm{t}\right) 3+ \\
& +T_{2}\left(\phi, x, z, \mathrm{t}^{2}+, \ldots \ldots+, \mathrm{o}\left(3^{k}\right.\right.
\end{aligned}
$$

Подставим (32) и (33) в (30), а также в (29). Затем приравняем нулю коэффициенты при $3^{k} k=0,1, \ldots$ т.д., в результате получим систему рекуррентных регулярных уравнений:

$$
\begin{aligned}
& 3^{-1}: \mathrm{c}\left(\frac{d \mathrm{n}_{x_{0}}}{d \phi}\right) s_{t}+\mathrm{c} \frac{d \mathrm{n}_{x_{0}}^{2}}{d \phi} S_{x}+\frac{\mathrm{cd}\left(\mathrm{n}_{x_{0}} \mathrm{n}_{z_{0}}\right)}{d \phi} S_{z}= \\
& =\frac{d^{2} \mathrm{n}_{x_{0}}}{d \phi^{2}}\left(s_{x}^{2}+s_{z}^{2}\right)+\frac{1}{3}\left(\frac{d^{2} \mathrm{n}_{x_{0}}}{d \phi^{2}} s_{x}^{2}+\frac{d^{2} \mathrm{n}_{z_{0}}}{d \phi^{2}} s_{x} s_{z}\right), \\
& \mathrm{c}\left(\frac{d \mathrm{n}_{z_{0}}}{d \phi}\right) s_{t}+\mathrm{c} \frac{d \mathrm{n}_{z_{0}}^{2}}{d \phi} S_{x}+\frac{\mathrm{cd}\left(\mathrm{n}_{x_{0}} \mathrm{n}_{z_{0}}\right)}{d \phi} S_{z}=
\end{aligned}
$$


$=\frac{d^{2} \mathrm{u}_{z_{0}}}{d \phi^{2}}\left(s_{x}^{2}+s_{z}^{2}\right)+\frac{1}{3}\left(\frac{d^{2} \mathrm{u}_{z_{0}}}{d \phi^{2}} s_{x}^{2}+\frac{d^{2} \mathrm{n}_{x_{0}}}{d \phi^{2}} s_{x} s_{z}\right)$,

$c_{p^{\prime}}$

$\mathrm{c} \frac{d m_{j 0}}{d \phi}\left(s_{t}+s_{x} \mathrm{U}_{x_{0}}+s_{z} \mathrm{U}_{z_{0}}=\mathrm{c} \frac{d^{2} m_{j 0}}{d \phi^{2}}\left(s_{x}^{2}+s_{z}^{2}\right.\right.$,

$\left.3^{0}: \mathrm{c}\left(\frac{d \mathrm{u}_{x_{1}}}{d \phi} s_{t}\right)+2 \mathrm{c} \frac{d}{d \phi}\right) s_{x}+\mathrm{c} \frac{d}{d \phi}\left(\mathrm{u}_{x_{1}} \mathrm{u}_{z_{0}}+\mathrm{u}_{z_{1}} \mathrm{u}_{x_{0}}\right) s_{z}=$

$\frac{d^{2} \mathrm{n}_{x_{1}}}{d \phi^{2}}\left(s_{x}^{2}+s_{z}^{2}\right)+$

$+\frac{1}{3}\left(\left(\frac{d^{2} \mathrm{u}_{x_{1}}}{d \phi^{2}} s_{x}^{2}+\frac{d^{2} \mathrm{u}_{z_{1}}}{d \phi^{2}} s_{x} s_{z}\right)+\frac{d \mathrm{u}_{x_{0}}}{d \phi} s_{x x}^{\prime \prime}+\frac{d \mathrm{u}_{z_{0}}}{d \phi} s_{z z}^{\prime \prime}\right)$

$\left.\mathrm{c}\left(\frac{d \mathrm{u}_{z_{1}}}{d \phi} s_{t}\right)+2 \mathrm{c} \frac{d}{d \phi}\right) s_{x}+\mathrm{c} \frac{d}{d \phi}\left(\mathrm{u}_{z_{1}} \mathrm{u}_{x_{0}}+\mathrm{u}_{x_{1}} \mathrm{u}_{z_{0}}\right) s_{z}=$

$\frac{d^{2} n_{z_{1}}}{d \phi^{2}}\left(s_{x}^{2}+s_{z}^{2}\right)+$

$\frac{1}{3}\left(\left(\frac{d^{2} \mathrm{u}_{z_{1}}}{d \phi^{2}} s_{x}^{2}+\frac{d^{2} \mathrm{u}_{x_{1}}}{d \phi^{2}} s_{x} s_{z}\right)+\frac{d \mathrm{u}_{x_{0}}}{d \phi} s_{x x}^{\prime \prime}+\frac{d \mathrm{u}_{z_{0}}}{d \phi} s_{z z}^{\prime \prime}\right)$,

$c_{p}=\frac{d^{2} T_{1}}{d \phi^{2}}\left(s \mid \chi^{2}+s_{z}^{2}\right)+\frac{d T_{0}}{d \phi}+s_{z z}^{\prime \prime}+R_{t_{1}}$

$\mathrm{c} \frac{d m_{j 1}}{d \phi}+\mathrm{c} \frac{d}{d \phi}\left(m_{j 1} \mathrm{~h}_{x_{0}}+\mathrm{u}_{x_{1}} m_{j 0}\right)=\frac{d^{2} m_{j 1}}{d \phi^{2}}\left(s_{x}^{2}+s_{z}^{2}+\right.$

$\left.\mathrm{c} \frac{d m_{j 1}}{d \phi}+s_{z z}^{\prime \prime}\right)+R_{t 0}$

$o(3):$

Данная система уже не будет, сингулярновозмущенной и ее можно будет последовательно решать известными методами.

\section{Выво $\triangle \mathrm{b}$}

В статье рассмотрена сингулярновозмущенная система оптимального управления с квадратичным критерием качества, доказан необходимый критерий оптимальности. Рассмотрен пример, демонстрирующий применение асимптотического метода регуляризации сингулярных задач оптимального управления, связан с уравнениями, описывающими процессы теплопередачи, массопередачи, химическими реакциями (процессы, лежащие в основании промышленного производства). Доказывается теорема 1, которая приводит сингулярную задачу оптимального управления к регулярному виду.

Далее, рассматривается аналогичное уравнение, однако, с возмущениями по координате. Здесь для регуляризации подобных задач применяется метод, аналогичный методу Фробениуса (теорема 2).

Следующий пример, использующий метод регуляризации, связан с моделированием процессов, протекающих в металлургических агрегатах. В статье рассмотрена сингулярновозмущенная задача оптимального управления, учитывающая баланс массы, импульса, а также тепла. С функционалом, который представляет наименьшее отклонение от заданного закона. Показано, что подобные распределенные сингулярновозмущенные задачи оптимального управления регуляризуются при помощи метода Уизема. Они приводятся к рекуррентной цепочке распределенных регулярных задач оптимального управления. К полученной цепочке можно уже достаточно просто применить численные методы.

Таким образом, в статье показывается, что наряду с сосредоточенными задачами можно развить метод регуляризации, применимый к распределенным сингулярновозмущенным задачам оптимального управления.

\section{Зак^ючение}

Результаты работы могут быть использованы в дальнейших теоретических и прикладных исследованиях $[11,12]$ сложных технологических процессов при создании многоуровневых систем управления, функционирующих в условиях дефицита информации.

\section{ЛИТЕРАТУРА}

1. Рутковский А.Л., Билаонов Б. Д., Ковалева М. А. Нерегулярно возмущенные процессы при горении топлива //В сборнике: Проблемы энергосбережения в промышленном и жилищно-коммунальном комплексах. Сборник трудов XV Международной научно-практической конференции. . 2014.—C. 208-214.

2. Рутковский А.Л., Арунянц Г. Г., Ковалева М. А., Тедеева Н. В. Формализация и решение задачи оптимального управления сложным технологическим комплексом // Вестник Балтийского федерального университета им. И. Канта. Серия: Физико-математические и технические науки. 2016. — № 1.—C. 81-89.

3. Рутковский А.Л., Ковалева М. А., Аликов А. Ю., Тедеева Н. В. Метод повышения эффективности расчета динамических характеристик объекта управления // Вестник Воронежского государственного университета. Серия: Системный анализ и информационные технологии. 2017. - № 2. - С. 16-21.

4. Рутковский А.Л., Ковалева М. А., Коробкин Р.С., Смольянинов В. В. Алгоритм адаптации параметров математической модели управления сложными нестационарными технологическими объектами// Естественные и технические науки. 2019. — № 6 (132). — C. 212-218.

5. Салихов 3.Г., Арунянц Г. Г., Рутковский А. Л. Системы оптимального управления сложными объектами. - М.: Теплотехника, 2004. - 496с.

6. Макаров И.М., Виноградская Т. М., Рубчинский А. А., Соколов В.Б. Теория выбора и принятия решений: Учебное пособие.- М.: Наука. Главная редакция физико-математической литературы, 1982.-328с. 
7. Попырин Л.С. Математическое моделирование и оптимизация теплоэнергетических установок. — M:1978. — 415 .

8. Левенталь Г.В., Попырин Л. С. Оптимизация теплоэнергетических установок. - Энергия, 1980. - 450с.

9. Бяринов А.М., Кафаров В. В. Методы оптимизации в химической технологии. — М.: Химия, 1975.— 575 с.

10. Звягин, Л.С. Системный анализ в экспериментальных исследованиях / Л.С. Звягин.— Текст: непосредственный // Молодой ученый. - $2014 .-$ № 4 (63).—C. 516-519.—URL: https://moluch.ru/archive/63/10108/

11. Rutkowski A.L., Bolotaeva I. I. and Kovaleva M. A. Modeling of Static Modes of Mass Transfer Processes in Rotary Kilns of Drum Type,// 2018 International Russian Automation Conference (RusAutoCon), Sochi, 2018, pp. 1-3.

12. Rutkowski A.L., Bolotaeva I. I. and Kovaleva M. A. Experiment Strategy and Parameter Evaluation at Fuzzy Measurement of Input Variables// 2019 International Conference on Industrial Engineering, Applications and Manufacturing (ICIEAM), Sochi, Russia, 2019, pp. 1-7.

( ) Ковалева Мария Александровна ( mary_kovaleva@list.ru).

Журнал «Современная наука: актуальные проблемы теории и практики»

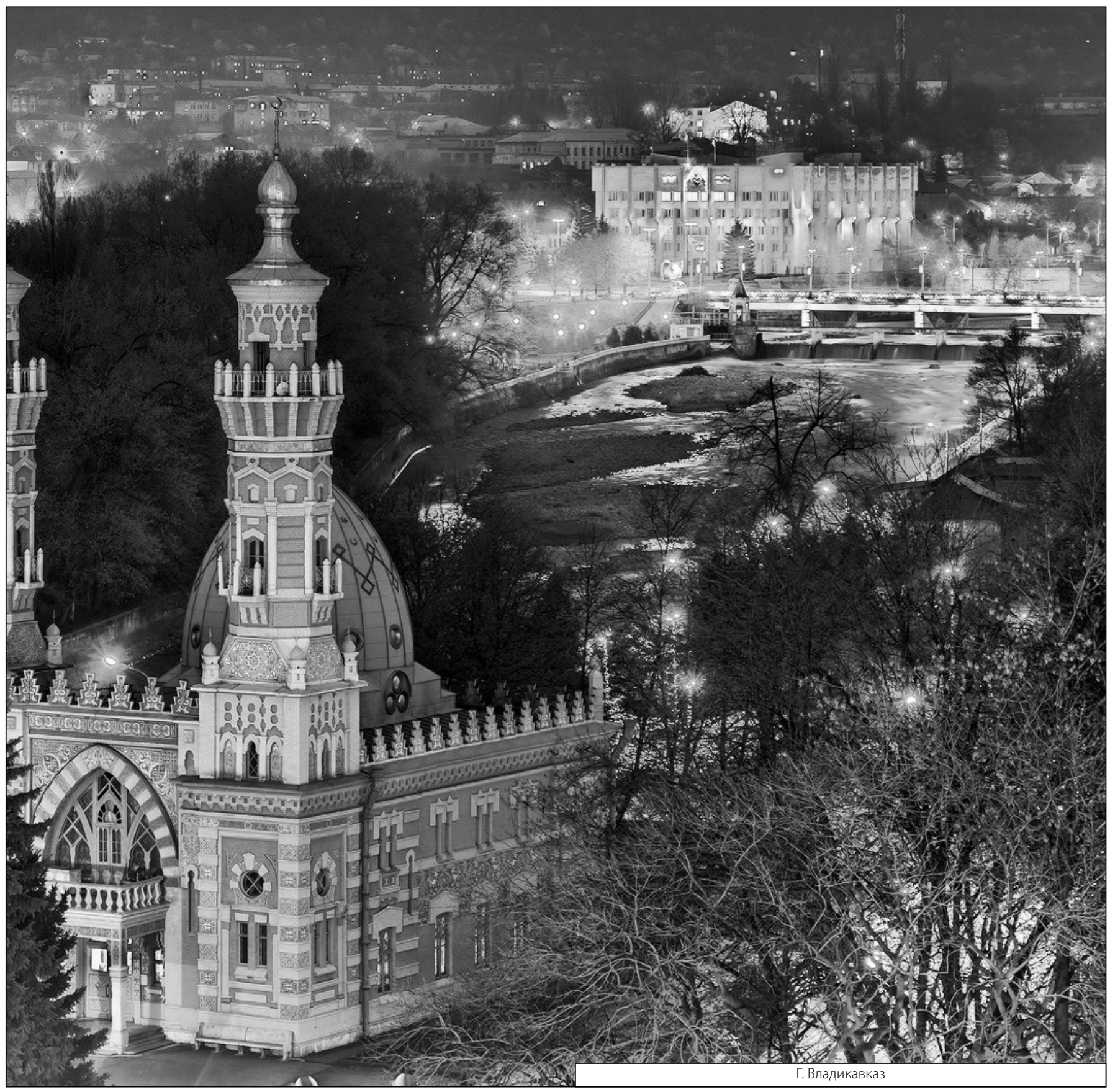

